\title{
Proteomics analysis of proteins interacting with heat shock factor 1 in squamous cell carcinoma of the cervix
}

\author{
LINGLI ZHANG ${ }^{1}$, ZHE HU $^{2}$, YING ZHANG ${ }^{1}$, JINZHI HUANG ${ }^{1}$, XUEFEN YANG $^{1}$ and JIAFENG WANG ${ }^{3}$ \\ Departments of ${ }^{1}$ Gynaecology and Obstetrics, ${ }^{2}$ Anesthesiology, and ${ }^{3}$ Stem Cell Research and Cellular Therapy Center, \\ Affiliated Hospital of Guangdong Medical University, Zhanjiang, Guangdong 524001, P.R. China
}

Received September 19, 2018; Accepted March 7, 2019

DOI: $10.3892 / \mathrm{ol} .2019 .10539$

\begin{abstract}
Protein interactions are crucial for maintaining homeostasis. Heat shock factor 1 (HSF1), a transcription factor that interacts with various proteins, is highly expressed in squamous cell carcinoma (SCC) of the cervix. The aim of the present study was to investigate the protein interaction profile of HSF1 in cervical SCC. Proteins interacting with HSF1 in SCC tissue and non-cancerous control (Ctrl) tissue were obtained by immunoprecipitation, separated by SDS-PAGE, identified by matrix-assisted laser desorption/ionization-time-of-flight mass spectrometry and analyzed using bioinformatics methods. A total of 220 and 241 proteins were identified by mass spectrometry in the tissues of Ctrl and SCC samples, respectively, among which 172 were detected exclusively in SCC (Pro-S), 151 exclusively in Ctrl (Pro-C) and 69 in both groups (Pro-B). The protein interaction profiles were different in each group; the STRING database identified three proteins that interacted with HSF1 directly, including insulin-like growth factor 1 receptor and small nuclear RNA-activating protein complex subunit 4 in Pro-C and small ubiquitin-related modifier 1 in Pro-S. Functional enrichment analysis of Gene Ontology revealed that the top terms were alternative splicing in Pro-S and polymorphism in Pro-C. In Pro-S, more categories were related to protein modification, such as phosphorylation, ubiquitination and acetylation. Therefore, HSF1 may influence the occurrence and development of cervical SCC by interacting with specific proteins.
\end{abstract}

Correspondence to: Dr Jiafeng Wang, Stem Cell Research and Cellular Therapy Center, Affiliated Hospital of Guangdong Medical University, 57 Renmin South Avenue, Xiashan, Zhanjiang, Guangdong 524001, P.R. China

E-mail: wangjiafeng_gz@163.com

Abbreviations: HSF1, heat shock factor 1; IP, immunoprecipitation; MS, mass spectrometry; SCC, squamous cell carcinoma of cervix

Key words: heat shock factor 1, protein interaction, squamous cell carcinoma of cervix, immunoprecipitation, mass spectrometry

\section{Introduction}

Cervical cancer is one of the most common malignant gynecological tumors, and squamous cell carcinoma of the cervix (SCC) is the most common histological subtype (1). According to a 2015 report by the Chinese Cancer Registry, cervical cancer has an incidence rate of 9.89 per 100,000 and a mortality rate of 2.6 per 100,000 (2). Further clarification of the molecular mechanisms underlying cervical cancer may lead to the development of improved treatment options for this disease.

Heat shock factor 1 (HSF1) was first identified as a classical transcriptional factor activated in the heat shock response (3). Upon activation by a variety of stimuli such as heat shock, infection and heavy metal toxicity, HSF1 forms trimers, translocates into the nucleus and induces various heat shock proteins (HSPs) by binding to heat shock elements (HSEs) on HSP promoters $(4,5)$. Accumulating evidence has suggested that HSF1 is a powerful modifier of carcinogenesis; HSF1 levels are elevated in several cancers, including breast (6), ovarian (7) and cervical cancer (8). Activated or elevated HSF1 is often associated with resistance to chemotherapy drugs or poor prognosis (9). A previous study revealed that $\mathrm{HSF}^{-1-}$ mice are resistant to chemically induced tumors, and that $\mathrm{HSF}^{-/}$mouse embryonic fibroblasts resist oncogene-induced transformation (10). However, the underlying mechanisms of the resistance remain to be determined.

Several studies have reported that HSF1 promotes a specific transcriptional program in highly malignant cells (11), as well as distinct transcriptional programs in cancer-associated fibroblasts and adjacent cancer cells (12). It is currently unknown why HSF1 regulates the transcription of different genes under different conditions. HSF1 has been reported to interact with numerous protein factors and serves multiple roles in various physiological and pathological processes, such as nuclear factor of interleukin 6 (13), 14-3-3 epsilon (14), heat shock transcription factor binding protein 1 (15) and 26S subunit, non-ATPase 10/gankyrin (16). Interactions with different proteins under various conditions may enable HSF1 to perform multiple functions. Therefore, clarifying the protein interaction profile of HSF1 in SCC may help identify the functions of HSF1.

In the present study, proteins that bind to HSF1 in the cervical tissues of patients with SCC and control subjects were obtained by using immunoprecipitation (IP) and separated by 
SDS-PAGE. The gel bands were analyzed by matrix-assisted laser desorption/ionization-time of flight mass spectrometry (MALDI-TOF-MS) to identify the proteins. Based on these results, interaction networks and potential molecular functions of the identified proteins were analyzed by bioinformatic methods.

\section{Materials and methods}

Patients and tissue samples. Cervical tissue samples were collected from four patients diagnosed with squamous cell carcinoma (SCC) of the cervix, who underwent total hysterectomy, as well as four control (Ctrl) patients diagnosed with hysteromyoma or adenomyosis with unaffected cervical tissue in Affiliated Hospital of Guangdong Medical University (Zhanjiang, China) between February and August 2013 (Table SI). All patients in the SCC group were not complicated with other types of pelvic cancer and did not receive chemoradiotherapy within 3 months before surgery. Disease staging was performed following the International Federation of Gynecology and Obstetrics (FIGO) classification. In addition, two groups met three major criteria: i) Higher HSF1 expression in SCC compared to Ctrl; ii) matched for age in the two groups $(43 \pm 2.94$ years in SCC; $45.25 \pm 3.69$ years in Ctrl); iii) similar clinical stage for SCC patients (three were in stage IIa and one in stage Ib2). The study was approved by the Medical Ethics Committee of the Affiliated Hospital of Guangdong Medical University (Zhanjiang, China; reference no. PJ2013046) and written informed consent was obtained from all patients before surgery. Patients were not treated with chemotherapy or radiotherapy for 3 months prior to surgery.

Protein extraction. Total protein from each sample (200 mg) was extracted in radioimmunoprecipitation assay (RIPA) lysis buffer (EMD Millipore, Billerica, MA, USA) plus $0.01 \%$ phenylmethylsulfonyl fluoride and protease/phosphatase inhibitor cocktail (Cell Signaling Technology, Inc., Danvers, MA, USA) in a homogenizer on ice, and insoluble debris was removed by centrifugation for $10 \mathrm{~min}$ at $10,800 \mathrm{x} \mathrm{g}$ at $4^{\circ} \mathrm{C}$. Protein concentrations were determined using a bicinchoninic acid assay kit (Beyotime Institute of Biotechnology, Beijing, China).

IP and electrophoresis. PureProteome protein G/A beads and a magnetic stand (EMD Millipore) were used to immunoprecipitate the proteins that bound to HSF1, following the manufacturer's protocol. Briefly, $100 \mu \mathrm{l} \mathrm{G/A}$ beads were incubated with rabbit polyclonal HSF1 antibody (1:50, cat. no. ab2923; Abcam, Cambridge, UK) at $25^{\circ} \mathrm{C}$ for $10 \mathrm{~min}$ with continuous mixing. Following washing with PBS containing $0.1 \%$ Tween 20 ( $\mathrm{pH} 7.4$ ), the immobilized capture antibody was incubated with pooled protein $(500 \mu \mathrm{g}$, in equal amounts from 4 samples) at $4^{\circ} \mathrm{C}$ overnight with continuous mixing. The immunoprecipitates were washed with PBS containing $0.1 \%$ Tween 20 ( $\mathrm{pH} 7.4$ ), eluted by boiling in RIPA buffer and resolved by $10 \%$ SDS-PAGE.

Gel staining. The gels were stained with Coomassie Brilliant Blue R250 (0.1\% R250, 40\% methanol, 10\% glacial acetic acid) at $25^{\circ} \mathrm{C}$ for $20 \mathrm{~min}$ and then washed 4 times with washing buffer (10\% methanol, 10\% glacial acetic acid) for 10 min each time. To avoid interference from heavy/light antibody chains in MS analysis, 10 pairs of bands distant from the antibody chains exhibiting differences between the SCC and Ctrl groups were cut from the gel.

In-gel digestion. In-gel digestions were prepared as described by Ostling et al (17). Briefly, gel sections were minced into $1 \mathrm{~mm}^{3}$ pieces and washed 4 times with $100 \mathrm{mM}$ ammonium bicarbonate $\left(\mathrm{NH}_{4} \mathrm{HCO}_{3}\right)$ in $50 \%$ acetonitrile $(\mathrm{ACN}$; Thermo Fisher Scientific, Waltham, MA, USA). The gel fragments were subjected to reduction using $10 \mathrm{mM}$ dithiothreitol (Sigma-Aldrich; Merck KGaA, Darmstadt, Germany) in $100 \mathrm{mM} \mathrm{NH} \mathrm{HCO}_{3}$ buffer at $60^{\circ} \mathrm{C}$ for $1 \mathrm{~h}$. Alkylation was performed in a solution of $55 \mathrm{mM}$ iodoacetamide (Sigma-Aldrich; Merck KGaA) in $100 \mathrm{mM} \mathrm{NH}_{4} \mathrm{HCO}_{3}$ for $30 \mathrm{~min}$ at $25^{\circ} \mathrm{C}$ in the dark followed by in-gel digestion with $10 \mu \mathrm{l}$ of trypsin (25 ng/ $\mu \mathrm{l}$; Promega Corporation, Madison, WI, USA) in $50 \mathrm{mM} \mathrm{NH}_{4} \mathrm{CO}_{3}$ at $37^{\circ} \mathrm{C}$ for $14-16 \mathrm{~h}$. Subsequently, the supernatant was transferred to a new microcentrifuge tube and $100 \mu \mathrm{l}$ of $60 \%$ ACN containing $0.1 \%$ trifluoroacetic acid (TFA; Sigma-Aldrich; Merck KGaA) was added to the remaining debris, which was then treated by sonication for $15 \mathrm{~min}$ to collect the supernatant at room temperature. The two parts of the supernatant were combined and vacuum-dried to a volume of $10 \mu \mathrm{l}$. The vacuum-dried supernatant was concentrated with a ZipTips ${ }^{\circledR} \mathrm{C} 18$ (EMD Millipore) and washed 3 times with $0.1 \%$ TFA. The samples were subsequently eluted with buffer $(0.1 \%$ TFA and 50\% ACN) and $1 \mu \mathrm{l} \alpha$-cyano-4-hydroxycinnamic acid $(5 \mathrm{mg} / \mathrm{ml})$ was added before spotting onto the target plate.

MALDI-TOF-MS. Peptide mass fingerprinting (PMF) and sequence analysis were carried out on a MALDI-TOF/TOF mass spectrometer (4800 Proteomics Analyzer; Applied Biosystems; Thermo Fisher Scientific, Inc.). Internal standard calibration of the PMF spectra was performed using the autolysis products of trypsin. Peptide mass maps were acquired in positive reflection mode at an accelerating voltage of $25 \mathrm{kV}$ and laser intensity of 6,000, averaging 1,200 laser shots per MALDI-TOF spectrum and 2,400 shots per TOF/TOF spectrum (the resolution was 20,000). The top 10 precursor ion mass peaks with a mass range of 800-4,000 Da were selected for tandem TOF/TOF analysis. Two analysis types, combined MS and MS/MS, were used to examine the human NCBInr (ftp.ncbi. nlm.nih.gov/blast/db/FASTA/nr.gz) and Swissprot (ftp://ftp. uniprot.org/pub/databases/uniprot/current_release/knowledgebase/complete/)databases using Mascot software (version 2.2; Matrix Science Ltd.) to identify proteins that bind to HSF1. Searches were performed to allow for carbamidomethylation, oxidation and a maximum of one missed trypsin cleavage. Peptide tolerance and MS/MS tolerance were both 0.2 Da. Confidently identified proteins had a statistically significant protein score $(\mathrm{P}<0.05$ based on combined MS and MS/MS spectra).

Bioinformatics analysis. The interactions of MS-identified proteins from SCC and Ctrl samples were preliminarily confirmed using the STRING (version 10.5) online database (https://string-db.org). Functional categories and the Gene Ontology (GO) terms biological process, cellular component 
Table I. Proteins binding to heat shock factor 1 identified by mass spectrometry.

\section{Set}

Pro-C (n=151)

Pro-S $(n=172)$

Pro-B $(\mathrm{n}=69)$

\begin{abstract}
ACP7, AEBP1, AGO3, AKNAD1, ANKLE1, ARIH2OS, ASPM, ATP5I, ATPAF1, BICDL2, C10orf90, C15orf56, C17orf58, C20orf96, C6orf223, C9orf3, CALCB, CALHM2, CATSPERG, CCT3, CCT8, CDK10, CEP250, CEP57, CEP97, CHD2, CHD8, CHST9, COL18A1, CPNE8, CSNK1G2-AS1, CSPP1, CXorf23, DAOA, DIP2C, DKFZP434L187, DNAH10, DNAH11, DNAH6, DPCD, DRP2, DSTYK, DUSP1, ECHDC1, FANK1, FAT2, FAT4, FAU, FMN2, FNIP1, FRA10AC1, GAB1, GLI3, GMFB, GOLGA4, HACE1, HECW1, HRG, IGF1R, IGHA1, IGHG4, IGHM, IGSF21, IL21, IPMK, IPPK, ITPR1, KCNH8, KDM2A, KIF13A, KLHL34, KRT10, KRT9, LDOC1, LOXL2, LRRC27, LTA, MCEE, MGAT3, MICALL2, MIR4697HG, MRPL49, MRPS7, MTCL1, MTIF2, MTO1, MX2, MYCBP, NEB, NEBL, NEU3, NR1I3, NRAP, NUP160, PCDHB15, PCDHGC4, PDCD7, PGK2, PHLDA3, PLEKHA5, PPARGC1A, PPP4R1, PRTG, PTPN23, RASSF3, RBM12B, RCAN2, RD3, RFC3, RFX2, RIPK2, RND1, RPL13, RPL36AL, RPS20, RPS28, RTL1, S100P, SBDS, SCAPER, SCGB1D2, SH2D1B, SHOX2, SLC15A2, SLC4A1AP, SMARCA5, SMC5, SNAPC4, SNX31, STAT4, TAF6, TAP1, TENM2, TLN2, TMEM266, TMEM59L, TP53TG1, TSGA13, TTN, TUBGCP2, TXNDC8, UBD, USP28, WFDC11, ZC2HC1B, ZC3H18, ZMYND10, ZNF148, ZNF536, ZNF770, ZNF837
\end{abstract}

AASS, ABCG4, ALK, ALPL, AMIGO2, AMPD3, AP2A1, AP3D1, ARHGAP32, ARHGEF18, ATN1, ATP13A4, ATP5G3, ATXN7, AURKC, BAHCC1, BCL2L11, BIRC6, BLZF1, BTN3A3, C7orf13, CAB39L, CACNA1H, CASP5, CCDC105, CCL17, CCT2, CFAP57, CHAT, CHRNB4, CIC, CLASP2, CNTN1, COA4, COL4A3BP, CTNNBIP1, CUL4B, CUX2, DDX41, DDX56, DEF8, DLEC1, DMWD, DNAJC22, DVL3, DYNC1LI1,ENO1, ENPP3, FAM46C, FBXO42, FDXACB1, FGFRL1, FHOD3, FLJ42569, FMNL2, FREM2, GAREM2, GEMIN4, GLRB, GLTP, GRAP, GUSB, HDAC11, HECW2, HELLS, HIST1H1T, HLA-DPA1, ICAM3, IGHV3-30, IGHV3-33, IKZF4, ITIH5, KAT2A, KDM2B, KDM5B, KIF14, KLHL25, KRR1, KRT16, LMNB1, LRP4, MAP4K3, MDH1B, MESP1, MRM1, MRPS18A, MS4A10, MYO15A, MYO1A, MYT1, MYT1L, NEDD8, NPC1L1, NPHP3, NRXN1, NSD1, NXF3, OR1S2, OR2T7, OR6T1, OTUD4, OTUD7A, PADI6, PATE2, PCNX3, PDGFRL, PDK4, PHB2, PHRF1, PKD1L1, PLXNB3, PNLIPRP3, PNN, PPP1R42, PRDX3, PRPF4, PSMB10, PSMB3, PSMC3, RALGDS, RBBP5, RCN3, RGL1, RHPN1, RIMKLA, RIMS2, RIPK4, RNF139, RNF168, RPGRIP1L, RTTN, RYR2, SAG, SERBP1, SETD1A, SH2B3, SH2D4B, SHISA7, SLC25A30, SLFN11, SMARCA5, SMIM21, SORD, SOWAHC, SPECC1L, SQRDL, SRRM1, ST20, STAR, SUMO1, TBC1D10C, TBC1D4, TENM1, TENM3, TERT, TESK2, TFAP4, TGM7, TMEM215, TNK1, TRABD, TSSK6, TXNRD2, VPS13A, WNT2B, YIF1A, ZDHHC2, ZFP64, ZNF185, ZNF438, ZNF506, ZNF790

ACSM3, ANKRD6, ARFGEF3, ASTE1, ATP5O, BCLAF1, BDP1, BRWD1, BTN3A2, C2CD2L, CCDC81, CCDC88A, CDKN2AIP, CLIP3, COX6A2, DIO3, DNAJC19, DPF1, DPH5, EFCAB10, GALP, GRIK2, GRIN1, HAX1, HSPE1, HYPK, IDH1, IFNA2, IGHA2, IGHG1, IGHG2, IGHG3, IGHM, IGHV2-70, IGHV3-23, IGKC, IL4, KRT1, MAST4, MBLAC2, MMEL1, NBR1, NPFFR1, NPHS1, PCBD2, PFDN6, PHKG1, POLL, POLR2G, PRL, PROSC, PTH2,PYDC1, RAB6B, RPAP1, RPL10, RYR3, SART1, SDR42E2, SLC46A3, SLIRP, SYT11, TAS2R14, TRIM32, UBE3D, UMPS, URI1, ZBTB10, ZNF292

Pro-B, proteins identified by mass spectrometry occurring in both groups; Pro-C, proteins identified by mass spectrometry in the control group only; Pro-S, proteins identified by mass spectrometry in the cervical squamous cell carcinoma group only.

and molecularfunction of MS-identified proteins were analyzed using the Database for Annotation, Visualization and Integrated Discovery (DAVID 6.8; https://david.ncifcrf.gov).

\section{Results}

Proteins binding to HSF1. In order to investigate the effect of HSF1, four SCC samples with higher HSF1 expression levels than the Ctrl samples were used in the current study (Fig. S1). A total of 392 different proteins were identified by MS, with 241 and 220 proteins in SCC and Ctrl samples, respectively. The proteins were distributed as follows: 172 proteins only occurred in SCC (termed Pro-S), 151 proteins were identified only in Ctrl (termed Pro-C), and 69 proteins were identified in the two groups (termed Pro-B) and were removed from further analysis (Table I). Furthermore, the immunoprecipitated proteins binding to HSF1 yielded a different SDS-PAGE electropherogram (Fig. S2).

Interaction analysis. The STRING website was used to determine whether the proteins identified by MS bound to HSF1. A total of 151 proteins from the Pro-C set were entered along with HSF1, which produced a network consisting of 144 nodes and 61 edges. In this network, HSF1 was indicated to interact 

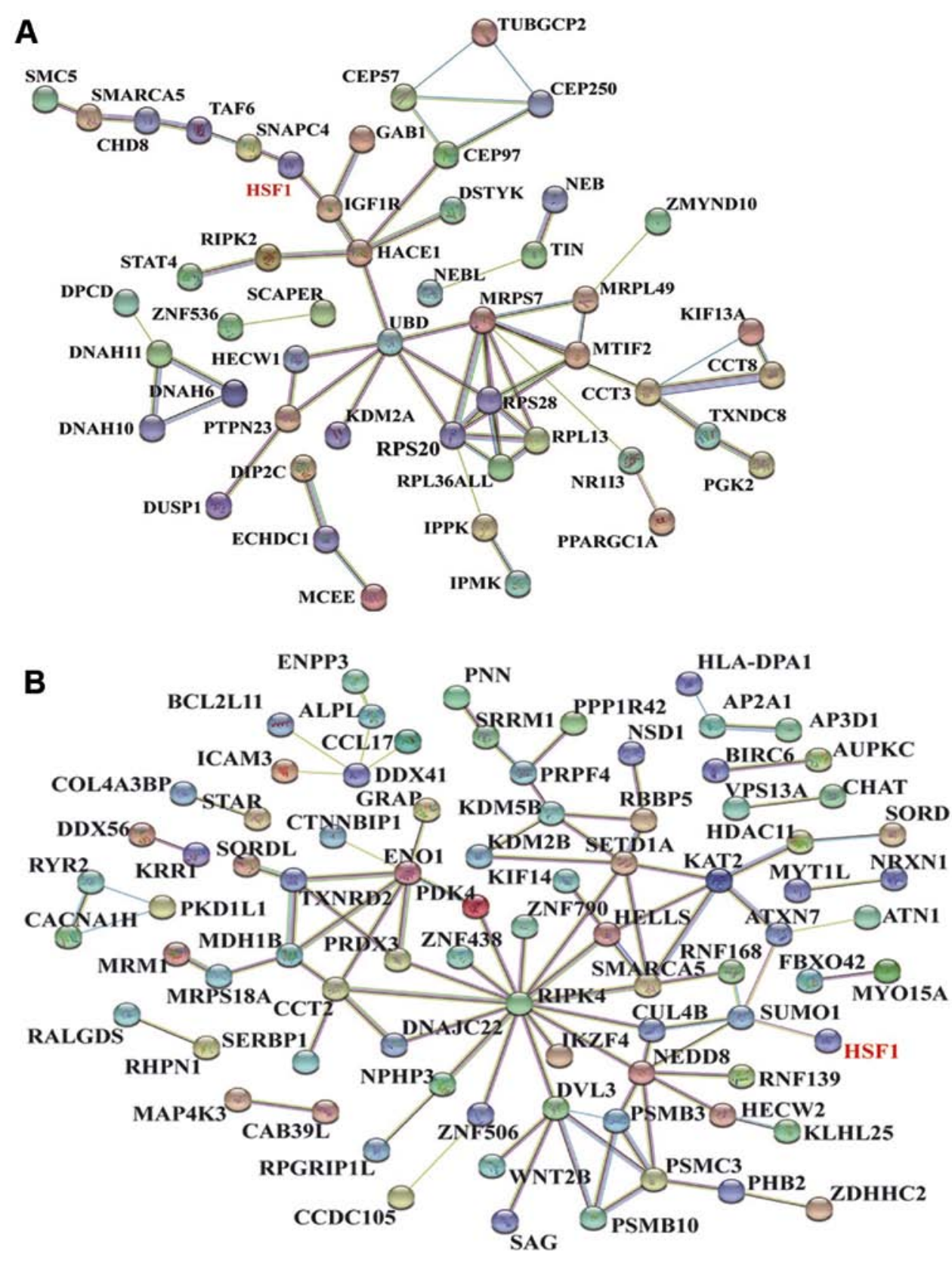

Figure 1. Interaction network predicted using the STRING website. (A and B) Interaction network of proteins identified by mass spectrometry found only in (A) Pro-C or (B) Pro-S. Pro-C, proteins in the control group only; Pro-S, proteins in the cervical squamous cell carcinoma group only.

with insulin-like growth factor 1 receptor and small nuclear RNA-activating protein complex subunit 4 ; these proteins interacted with others to form a highly complex network (Fig. 1A).

HSF1 and the 172 proteins from the Pro-S set were also entered, and a network consisting of 167 nodes and 92 edges was obtained. The results identified an interaction between HSF1 and small ubiquitin-related modifier 1 (SUMO1). Additionally, SUMO1 was indicated to interact with other proteins to construct a highly complicated network, which included neural precursor cell-expressed developmentally downregulated protein 8 (NEDD8), cullin-4B, receptor-interacting serine-threonine kinase 4 (RIPK4), and disheveled segment polarity protein 3 (DVL3) from the Pro-S set (Fig. 1B).

Functional classification. Subsequently, 151 proteins from the Pro-C set and 172 proteins from the Pro-S set were entered into DAVID bioinformatics resources. Two of these proteins, immunoglobulin heavy constant $\mu$ and caspase 5, were not recognized by the database and removed from further analysis. Therefore, 150 proteins from Pro-C and 171 proteins from Pro-S were subjected to functional classification according to the UniProtKB Keywords original database in DAVID. Following this analysis, 130/150 proteins from Pro-C and 147/171 proteins from Pro-S were present in the output. The results revealed the distribution of Pro-S proteins into functional categories that differed from those of Pro-C (Fig. 2). The predominant category in Pro-S was 'alternative splicing' (57.31\%), whereas the largest category in Pro-C was 'polymorphism' (70.00\%). 'Phosphoprotein' was the second largest category in both groups, representing $55.56 \%$ of proteins in Pro-S and $48.00 \%$ of proteins in Pro-C. The third largest category was related to cellular localization in both sets; however, 
A

B
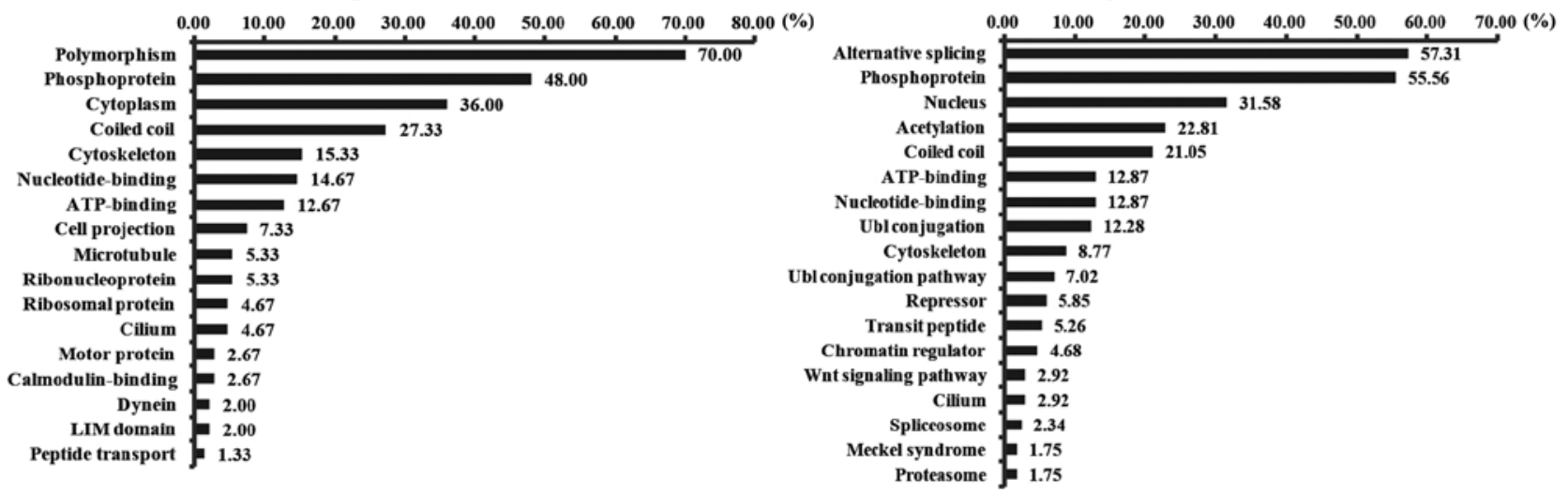

Figure 2. Functional classification of proteins identified by MS. (A and B) Functional classification of proteins identified by MS as binding to HSF1 in (A) Pro-C or (B) Pro-S. HSF1, heat shock factor; MS, mass spectrometry; Pro-C, proteins in the control group only; Pro-S, proteins in the cervical squamous cell carcinoma group only.

this category was 'nuclear localization' (31.58\%) in Pro-S and 'cytoplasmic localization' (36.00\%) in Pro-C. Furthermore, five categories were present in the two groups at comparable frequencies: 'Coiled coil', 'ATP binding', 'nucleotide binding', 'cytoskeleton' and 'cilium'. A considerable fraction of the proteins indicated to bind HSF1 in Pro-S were associated with protein modifications, including 'acetylation' (22.81\%), 'ubiquitin-like (Ubl) conjugation' (12.28\%) and 'Ubl conjugation pathway' (7.02\%).

GO term analysis. The protein sets obtained for Pro-S and Pro-C were subjected to GO term analysis. For the cellular component category, 79/150 proteins in Pro-C and 82/171 proteins in Pro-S were identified. The results revealed that the proteins were mainly located in the cytoplasm and nucleoplasm in Pro-C, whereas the proteins were in the cytosol, nucleoplasm and membrane in Pro-S (Fig. 3A). For the molecular function category, 49/150 proteins in Pro-C and 31/171 proteins in Pro-S were analyzed. The largest category was 'ATP binding' in the two sets and the category 'poly (A) RNA binding' also has large proportion in Pro-C (Fig. 3B). For the biological process category, 39/150 proteins in Pro-C and 51/171 proteins in Pro-S were analyzed. The largest two categories were 'protein phosphorylation' and 'negative regulation of the canonical Wnt signaling pathway' in Pro-S, and 'translation' and 'positive regulation of apoptotic process' in Pro-C (Fig. 3C).

\section{Discussion}

HSF1 may bind to distinct proteins in SCC. Tumorigenesis is a complex process involving various protein factors and molecular pathways. HSF1 is a transcription factor involved in multiple diseases through interactions with numerous proteins, including nuclear factor-interleukin-6 and 14-3-3e $(14,18)$. This is the first study, to the best of our knowledge, to examine the function of HSF1 at the interactional proteome level in SCC. Clear differences in the proteins that bind to HSF1 were observed between cervical SCC tissue and Ctrl tissue, which indicated that the proteins binding to HSF1 were distinct in the two tissue types.
HSF1 may participate in alternative splicing in SCC. The proteins identified by MS as only present in SCC or Ctrl tissues were classified using the online functional annotation tool DAVID. The largest category of proteins binding to HSF1 in Pro-S were involved in alternative splicing. Alternative splicing enables the production of different combinations of exons from the same genomic template and therefore increases protein complexity (19). In cervical cancer, alternative splicing of numerous genes, including Numb (20), survivin Dex3 (21) and RAB1B, member RAS oncogene family (22), has been reported. Additionally, the serine/arginine rich family of proteins dictate splice site recognition (23). HSF1 is a serine-rich protein (24), which suggests that HSF1 may interact with certain proteins to participate in alternative splicing of genes in SCC. However, the association between HSF1, alternative splicing and cervical cancer has not been widely examined.

HSF1 may have unique phosphorylation in SCC. Phosphoproteins was the second largest category in Pro-C and Pro-S. HSF1 is a serine-rich constitutively phosphorylated mediator of the stress response (24) and can be phosphorylated at multiple sites, including Ser230 (25), Ser303 and Ser307 (26). The inducible phosphorylation of HSF1 is correlated with its transcriptional activation (27). piR-823 may influence whether HSF1 phosphorylation functions as a tumor promoter (28). Therefore, HSF1 phosphorylation status may serve a role in the genesis and progression of cervical cancer. Among the phosphoproteins in Pro-S, two common kinases were detected: RIPK4 and mitogen-activated protein kinase kinase kinase kinase 3 (MAP4K3; Table I). RIPK4 functions as a node in the protein network for the Pro-S set. Increased RIPK4 expression is associated with the progression and favorable prognosis of cervical cancer (29); RIPK4 promotes canonical Wnt signaling by phosphorylating disheveled segment polarity protein 2 (30). HSF1 Ser326 is a substrate of p38 mitogen-activated protein kinases (31). These data suggested that distinct kinases may be activated and interact with HSF1 in cervical cancer.

SUMO1 was identified in the 'alternative splicing' and 'phosphoproteins' categories (data not shown) and was confirmed by String to interact with HSF1. SUMO1 was 
A a

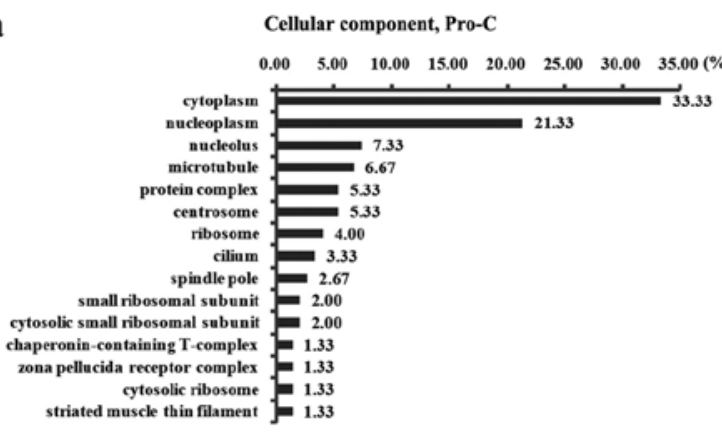

B a

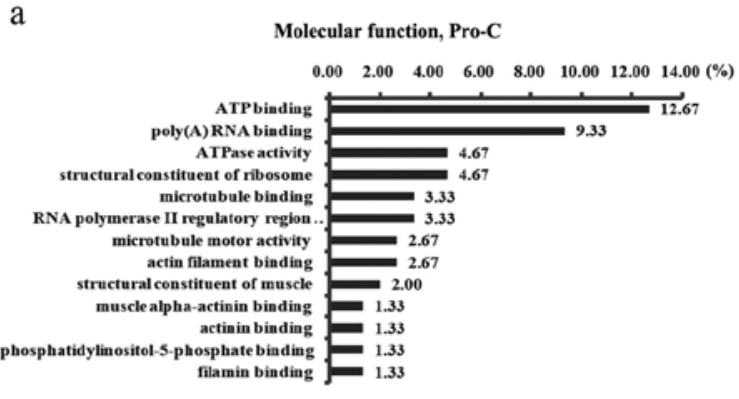

$\mathrm{C}$ a

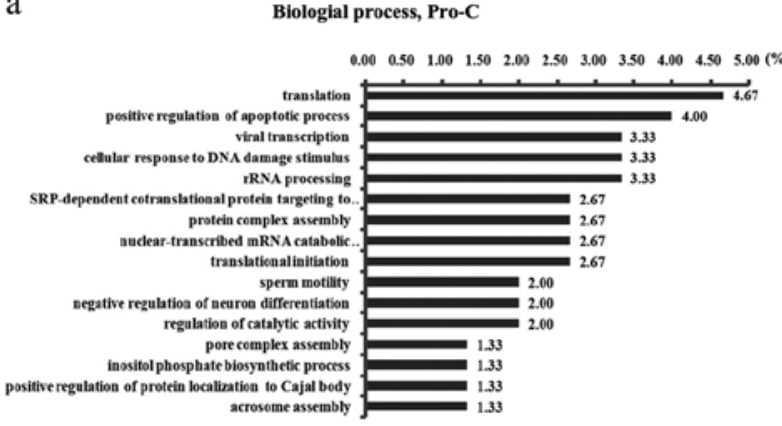

b

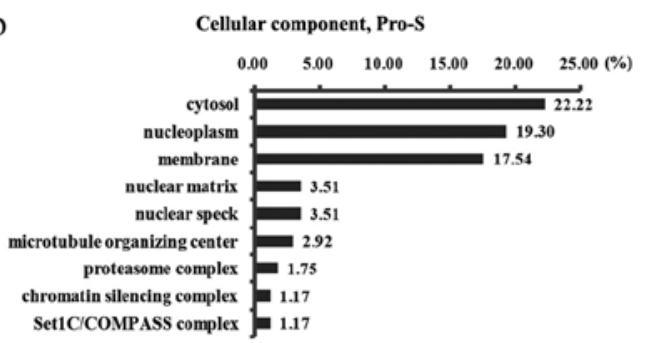

b

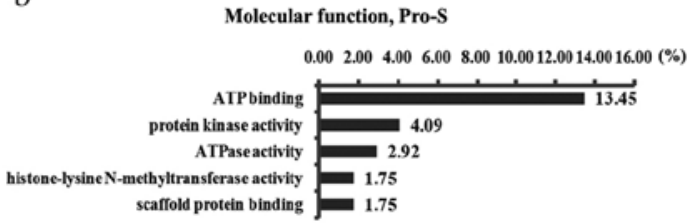

b

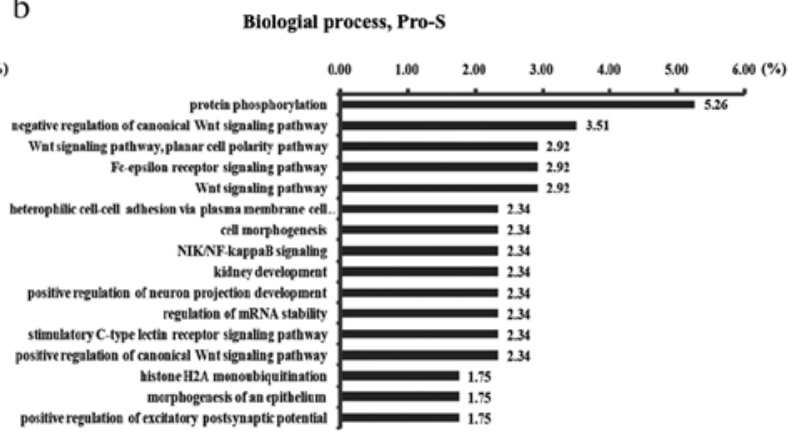

Figure 3. GO term analysis of proteins identified by MS. (A) The cellular component, (B) molecular function and (C) biological process of GO analysis. GO analysis of proteins occurred only in (A-a, B-a, C-a) Pro-C or (A-b, B-b, C-b) Pro-S. GO, Gene Ontology; HSF1, heat shock factor 1; MS, mass spectrometry; Pro-C, proteins in the control group only; Pro-S, proteins in the cervical squamous cell carcinoma group only.

demonstrated to regulate HSF1 DNA-binding activity through sumoylation at Lys298 (32). No previously published studies have investigated the correlation of SUMO1, HSF1 and cervical cancer. Therefore, whether the interaction between SOMU1 and HSF1 is involved in the occurrence and development of cervical cancer deserves attention.

HSF1 may exhibit greater translocation into the nucleus in SCC. The third largest functional category was 'nucleus' in Pro-S and 'cytoplasm' in Pro-C. Although GO cellular component analysis revealed that the cytoplasm was the largest and nucleoplasm the second largest term in both Pro-S and Pro-C, the ratio of protein in the nucleoplasm vs. cytoplasm was higher in Pro-S (0.87) compared with in Pro-C (0.64). HSF1 is activated and translocates from the cytoplasm into the nucleus under stress conditions (4). The results of the present study indicated that more HSF1 translocated to the nucleus in cervical SCC compared with normal tissue. Therefore, blocking the translocation of HSF1 during malignant transformation, for example, in human papilloma virus infection, may represent a therapeutic strategy.

In the molecular function GO analysis, 'ATP binding' was the largest category in Pro-C and Pro-S. In this category, dynein light intermediate chain 1 (DYNC1LI1) was identified in ATP binding and nucleotide binding categories (data not shown). DYNC1LI1 is a transporter in eukaryotic cells that serves important roles in the development and function of the mammalian nervous system (33) and malignancy of glioma (34). HSF1 has been reported to reinforce cell death resistance in glioma (35). The translocation of HSF1 under stress is not well-understood; further studies of the role of DYNC1LI1 in SCC may clarify the mechanism of HSF1 translocation.

HSF1 may influence acetylation in SCC. The fourth largest functional category in the Pro-S group was acetylation. Acetylation introduces an acetyl functional group into a chemical compound, which is an important modification of proteins at co-translational and post-translational level. Studies of histone acetylation in cervical cancer have revealed that histone $\mathrm{H} 3$ acetyl $\mathrm{K} 9$ was correlated with low grading and low FIGO staging scores (36). Col et al reported that HSF1 regulates the acetylation of pericentric chromatin such as histone H3K9 or H3K4 under heat stress (37). These data suggested that HSF1 may promote the development of cervical cancer by influencing the acetylation of H3K9. 
HSF1 may bind to a distinct ubiquitin protein ligase in SCC. The fifth largest functional category in the Pro-S group was 'coiled coil'. Coiled coil is a structural motif in proteins in which 2-7 a-helices are coiled together resembling strands of a rope (38). In the present study, two coiled-coil proteins were identified in the same E3 ubiquitin protein ligase family: HECT, $\mathrm{C} 2$ and WW domain-containing E3 ubiquitin protein ligase (HECW) 1 and HECW2; HECW1 was identified in Pro-C and HECW2 was identified in Pro-S (Table I). HECW1 interacts with RNF43 to enhance pro-apoptotic activity through p53 (39). HECW2 enhances endothelial cell-to-cell junctions, and its deficiency impairs angiogenesis (40). This suggested that HSF1 may interact with different members of the E3 ubiquitin protein ligase family under pathological and normal conditions.

HSF1 and Wnt signaling pathway. Although the Wnt signaling pathway appeared very low in the functional categories $(2.92 \%$, Fig. 2B), the biological process related to Wnt signaling pathway appeared four times in GO analysis for Pro-S (Fig. 3C b). The reason may be there were fewer members of this pathway. Wnt signaling pathway is involved in the development and metastasis of cervical cancer $(41,42)$. HSF1 increases Wnt/ $\beta$-catenin pathway activation (43), which suggested that HSF1 may be involved in the carcinogenesis of cervical cancer by regulating the Wnt signaling pathway. Interestingly, in the present study, the biological processes included not only positive but also negative regulation of canonical Wnt signaling pathway. It is possible that HSF1 may increase Wnt pathway activation indirectly by antagonizing the effect of a protein which can negative regulate the Wnt signaling pathway. The biological process of 'negative regulation of the canonical Wnt signaling pathway' included six proteins: Proteasome subunit $\beta$ (PSMB) 10; nephrocystin 3; DVL3; low-density lipoprotein receptor-related protein 4; PSMB3; and the proteasome 26S subunit, ATPase 3 (PSMC3) (data not shown). Among these proteins, PSMB10, PSMB3, PSMC3 and DVL3 formed a quadrilateral network as part of a larger network with HSF1 through NEDD8 and SUMO1 (Fig. 1B). Further studies are needed to explore whether HSF1 is able to regulate the Wnt signaling pathway by binding to this protein.

Although SUMO1 was the only protein identified in Pro-S and interacting with HSF1 directly (Fig. 1B), the results of the present study indicated possible indirect interactions between HSF1 and numerous proteins in the context of cervical cancer. The current study provided insight into the mechanism by which HSF1 influences the development of cervical cancers. However, owing to its preliminary nature, the study has some deficiencies such as small sample size and incomprehensive selection of reagents. For example, the RIPA buffer may disrupt weak non-covalent protein-protein interactions. In addition, we only chose 10 paired bands with obvious difference for MS. All these may possibly result in certain proteins, which have been reported as interactional proteins of HSF1, not being identified. Thus, future studies need to be conducted to confirm these interactions and to explore their functions in cervical SCC.

\section{Acknowledgements}

Not applicable.

\section{Funding}

This study was funded by The National Natural Science Foundation of China (grant nos. 81302246 and 81600445), The Start-up Fund for Doctors of Guangdong Medical University, China (grant no. B2012038) and The Support Fund for Key Discipline of Guangdong Medical University, China (grant no. 301K20140002).

\section{Availability of data and materials}

All data used and/or analyzed during the current study are available from the corresponding author upon reasonable request.

\section{Authors' contributions}

LZ contributed to design and drafting the manuscript. $\mathrm{ZH}$ analyzed the data of mass spectrometry. YZ analyzed and interpreted the patients' data. JH conducted IP. XY conducted protein extraction and electrophoresis. JW performed the bioinformatics analysis and gave final approval of the version to be published. All authors read and approved the final manuscript.

\section{Ethics approval and consent to participate}

This study was approved by The Medical Ethics Committee of Affiliated Hospital of Guangdong Medical University (Zhanjiang, China; reference no. PJ2013046) and all patients provided informed written consent.

\section{Patient consent for publication}

Not applicable.

\section{Competing interests}

The authors declare that they have no competing interests.

\section{References}

1. Hsu HC, Li X, Curtin JP, Goldberg JD and Schiff PB: Surveillance epidemiology and end results analysis demonstrates improvement in overall survival for cervical cancer patients treated in the era of concurrent chemoradiotherapy. Front Oncol 5: 81, 2015.

2. Chen W, Zheng R, Baade PD, Zhang S, Zeng H, Bray F, Jemal A, Yu XQ and He J: Cancer statistics in China, 2015. CA Cancer J Clin 66: 115-132, 2016.

3. Ritossa F: A new puffing pattern induced by temperature shock and DNP in Drosophila. Experientia 18: 571-573, 1962.

4. Baler R, Dahl G and Voellmy R: Activation of human heat shock genes is accompanied by oligomerization, modification, and rapid translocation of heat shock transcription factor HSF1. Mol Cell Biol 13: 2486-2496, 1993.

5. Shi Y, Mosser DD and Morimoto RI: Molecular chaperones as HSF1-specific transcriptional repressors. Genes Dev 12: 654-666, 1998.

6. Santagata S, Hu R, Lin NU, Mendillo ML, Collins LC, Hankinson SE, Schnitt SJ, Whitesell L, Tamimi RM, Lindquist S and Ince TA: High levels of nuclear heat-shock factor 1 (HSF1) are associated with poor prognosis in breast cancer. Proc Natl Acad Sci USA 108: 18378-18383, 2011.

7. Powell CD, Paullin TR, Aoisa C, Menzie CJ, Ubaldini A and Westerheide SD: The heat shock transcription factor hsf1 induces ovarian cancer epithelial-mesenchymal transition in a 3D spheroid growth model. PLoS One 11: e0168389, 2016. 
8. Yu S, Cheng M, Hou L, Zhang W, Liu B and Linghu H: HSF1 expression in cervical carcinoma and its correlation with clinical pathological characteristics and prognosis. J Third Mil Med Univ 36: 560-563, 2017 (In Chinese).

9. Calderwood SK: Elevated levels of HSF1 indicate a poor prognosis in breast cancer. Future Oncol 8: 399-401, 2012.

10. Dai C, Whitesell L, Rogers AB and Lindquist S: Heat shock factor 1 is a powerful multifaceted modifier of carcinogenesis. Cell 130: 1005-1018, 2007.

11. Mendillo ML, Santagata S, Koeva M, Bell GW, Hu R, Tamimi RM, Fraenkel E, Ince TA, Whitesell L and Lindquist $S$ : HSF1 drives a transcriptional program distinct from heat shock to support highly malignant human cancers. Cell 150: 549-562, 2012.

12. Scherz-Shouval R, Santagata S, Mendillo ML, Sholl LM, Ben-Aharon I, Beck AH, Dias-Santagata D, Koeva M, Stemmer SM, Whitesell $\mathrm{L}$ and Lindquist $\mathrm{S}$ : The reprogramming of tumor stroma by HSF1 is a potent enabler of malignancy. Cell 158: 564-578, 2014.

13. Zhang L, Yang M, Wang Q, Liu M, Liang Q, Zhang $\mathrm{H}$ and Xiao X: HSF1 regulates expression of G-CSF through the binding element for NF-IL6/CCAAT enhancer binding protein beta. Mol Cell Biochem 352: 11-17, 2011.

14. Wang X, Grammatikakis N, Siganou A, Stevenson MA and Calderwood SK: Interactions between extracellular signal-regulated protein kinase 1, 14-3-3epsilon, and heat shock factor 1 during stress. J Biol Chem 279: 49460-49469, 2004.

15. Eroglu B, Min JN, Zhang Y, Szurek E, Moskophidis D, Eroglu A and Mivechi NF: An essential role for heat shock transcription factor binding protein 1 (HSBP1) during early embryonic development. Dev Biol 386: 448-460, 2014.

16. Luo T, Fu J, Xu A, Su B, Ren Y, Li N, Zhu J, Zhao X, Dai R, Cao J, et al: PSMD10/gankyrin induces autophagy to promote tumor progression through cytoplasmic interaction with ATG7 and nuclear transactivation of ATG7 expression. Autophagy 12: 1355-1371, 2016.

17. Ostling P, Björk JK, Roos-Mattjus P, Mezger V and Sistonen L: Heat shock factor 2 (HSF2) contributes to inducible expression of hsp genes through interplay with HSF1. J Biol Chem 282: 7077-7086, 2007.

18. Xie Y, Chen C, Stevenson MA, Auron PE and Calderwood SK: Heat shock factor 1 represses transcription of the IL-1beta gene through physical interaction with the nuclear factor of interleukin 6. J Biol Chem 277: 11802-11810, 2002.

19. Wang ET, Sandberg R, Luo S, Khrebtukova I, Zhang L, Mayr C, Kingsmore SF, Schroth GP and Burge CB: Alternative isoform regulation in human tissue transcriptomes. Nature 456: 470-476, 2008.

20. Rong C, Feng Y and Ye Z: Notch is a critical regulator in cervical cancer by regulating Numb splicing. Oncol Lett 13: 2465-2470, 2017.

21. Gaytan-Cervantes J, Gonzalez-Torres C, Maldonado V, Zampedri C, Ceballos-Cancino G and Melendez-Zajgla J: Protein Sam68 regulates the alternative splicing of survivin DEx3. J Biol Chem 292: 13745-13757, 2017.

22. Nikoshkov A, Broliden K, Attarha S, Sviatoha V, Hellström AC, Mints $\mathrm{M}$ and Andersson S: Expression pattern of the PRDX2, RAB1A, RAB1B, RAB5A and RAB25 genes in normal and cancer cervical tissues. Int J Oncol 46: 107-112, 2015.

23. Bradley T, Cook ME and Blanchette M: SR proteins control a complex network of RNA-processing events. RNA 21: 75-92, 2015.

24. Xia W, Guo Y, Vilaboa N, Zuo J and Voellmy R: Transcriptional activation of heat shock factor HSF1 probed by phosphopeptide analysis of factor 32P-labeled in vivo. J Biol Chem 273 8749-8755, 1998

25. Holmberg CI, Hietakangas V, Mikhailov A, Rantanen JO, Kallio M, Meinander A, Hellman J, Morrice N, MacKintosh C, Morimoto RI, et al: Phosphorylation of serine 230 promotes inducible transcriptional activity of heat shock factor 1. EMBO J 20: 3800-3810, 2001.

26. Chu B, Soncin F, Price BD, Stevenson MA and Calderwood SK: Sequential phosphorylation by mitogen-activated protein kinase and glycogen synthase kinase 3 represses transcriptional activation by heat shock factor-1. J Biol Chem 271: 30847-30857, 1996.
27. Cotto JJ, Kline M and Morimoto RI: Activation of heat shock factor 1 DNA binding precedes stress-induced serine phosphorylation. Evidence for a multistep pathway of regulation. J Biol Chem 271: 3355-3358, 1996.

28. Yin J, Jiang XY, Qi W, Ji CG, Xie XL, Zhang DX, Cui ZJ, Wang CK, Bai Y, Wang J and Jiang HQ: piR-823 contributes to colorectal tumorigenesis by enhancing the transcriptional activity of HSF1. Cancer Sci 108: 1746-1756, 2017.

29. Azizmohammadi S, Azizmohammadi S, Safari A Kaghazian M, Sadrkhanlo M, Behnod V and Seifoleslami M: High-Level Expression of RIPK4 and EZH2 contributes to lymph node metastasis and predicts favorable prognosis in patients with cervical cancer. Oncol Res 25: 495-501, 2017.

30. Huang X, McGann JC, Liu BY, Hannoush RN, Lill JR, Pham V, Newton K, Kakunda M, Liu J, Yu C, et al: Phosphorylation of Dishevelled by protein kinase RIPK4 regulates Wnt signaling. Science 339: 1441-1445, 2013.

31. Dayalan Naidu S, Sutherland C, Zhang Y, Risco A, de la Vega L, Caunt CJ, Hastie CJ, Lamont DJ, Torrente L, Chowdhry S, et al: Heat shock factor 1 Is a substrate for p38 mitogen-activated protein kinases. Mol Cell Biol 36: 2403-2417, 2016.

32. Hietakangas V, Ahlskog JK, Jakobsson AM, Hellesuo M, Sahlberg NM, Holmberg CI, Mikhailov A, Palvimo JJ, Pirkkala L and Sistonen L: Phosphorylation of serine 303 is a prerequisite for the stress-inducible SUMO modification of heat shock factor 1. Mol Cell Biol 23: 2953-2968, 2003.

33. Banks GT, Haas MA, Line S, Shepherd HL, Alqatari M, Stewart S, Rishal I, Philpott A, Kalmar B, Kuta A, et al: Behavioral and other phenotypes in a cytoplasmic Dynein light intermediate chain 1 mutant mouse. J Neurosci 31: 5483-5494, 2011.

34. Szeliga M, Obara-Michlewska M, Matyja E, Łazarczyk M, Lobo C, Hilgier W, Alonso FJ, Márquez J and Albrecht $\mathrm{J}$ : Transfection with liver-type glutaminase cDNA alters gene expression and reduces survival, migration and proliferation of T98G glioma cells. Glia 57: 1014-1023, 2009.

35. Antonietti P, Linder B, Hehlgans S, Mildenberger IC, Burger MC Fulda S, Steinbach JP, Gessler F, Rödel F, Mittelbronn M and Kögel D: Interference with the HSF1/HSP70/bag3 pathway primes glioma cells to matrix detachment and $\mathrm{BH} 3$ mimetic-induced apoptosis. Mol Cancer Ther 16: 156-168, 2017.

36. Beyer S, Zhu J, Mayr D, Kuhn C, Schulze S, Hofmann S, Dannecker C, Jeschke U and Kost BP: Histone H3 acetyl K9 and histone $\mathrm{H} 3$ tri methyl $\mathrm{K} 4$ as prognostic markers for patients with cervical cancer. Int J Mol Sci 18: pii: E477, 2017.

37. Col E, Hoghoughi N, Dufour S, Penin J, Koskas S, Faure V, Ouzounova M, Hernandez-Vargash H, Reynoird N, Daujat S, et al: Bromodomain factors of BET family are new essential actors of pericentric heterochromatin transcriptional activation in response to heat shock. Sci Rep 7: 5418, 2017.

38. Liu J, Zheng Q, Deng Y, Cheng CS, Kallenbach NR and Lu M: A seven-helix coiled coil. Proc Natl Acad Sci USA 103: $15457-15462,2006$.

39. Shinada K, Tsukiyama T, Sho T, Okumura F, Asaka M and Hatakeyama S: RNF43 interacts with NEDL1 and regulates p53-mediated transcription. Biochem Biophys Res Commun 404: 143-147, 2011.

40. Choi KS, Choi HJ, Lee JK, Im S, Zhang H, Jeong Y, Park JA, Lee IK, Kim YM and Kwon YG: The endothelial E3 ligase HECW2 promotes endothelial cell junctions by increasing AMOTL1 protein stability via K63-linked ubiquitination. Cell Signal 28: 1642-1651, 2016

41. Bahrami A, Hasanzadeh M, ShahidSales S, Yousefi Z, Kadkhodayan S, Farazestanian M, Joudi Mashhad M, Gharib M, Mahdi Hassanian S and Avan A: Clinical significance and prognosis value of Wnt signaling pathway in cervical cancer. J Cell Biochem 118: 3028-3033, 2017.

42. Zhang R, Lu H, Lyu YY, Yang XM, Zhu LY, Yang GD, Jiang PC, Re Y, Song WW, Wang JH, et al: E6/E7-P53-POU2F1-CTHRC1 axis promotes cervical cancer metastasis and activates Wnt/PCP pathway. Sci Rep 7: 44744, 2017.

43. Li G, Song Y, Zhang Y, Wang H and Xie J: miR-34b Targets HSF1 to suppress cell survival in acute myeloid leukemia. Oncol Res 24: 109-116, 2016. 